\title{
Medo de quedas e força muscular do quadril em idosos independentes da comunidade
}

\author{
Fear of falling and hip muscular strength in independent older adults of the community \\ Miedo a caerse y fuerza muscular de cadera en ancianos independientes de la comunidad \\ Larissa Bocarde', Jaqueline Mello Porto², Renato Campos Freire Júnior ${ }^{3}$, Júlia Adorno Fernandes 4 , \\ Ana Paula Midori Nakaishi ${ }^{5}$, Daniela Cristina Carvalho de Abreu ${ }^{6}$
}

\begin{abstract}
RESUMO I O medo de quedas pode fazer com que o idoso desenvolva estratégias que alteram o equilíbrio semiestático e dinâmico, predispondo-o a um risco aumentado de cair. A função muscular dos abdutores e adutores de quadril tem importante papel na manutenção da estabilidade postural. Entretanto, não se sabe se idosos com medo de cair apresentam maior comprometimento na função muscular do quadril. Assim, o objetivo foi comparar o pico de torque (PT) isométrico dos músculos abdutores e adutores de quadril entre idosos com e sem medo de quedas. Os participantes foram divididos em dois grupos: com $(n=81)$ e sem $(n=81)$ medo de quedas. O PT dos abdutores e adutores de quadril foi obtido com dinamômetro isocinético (System 4 Pro, Biodex, Nova York, EUA). A comparação do PT dos grupos foi realizada por meio de modelo linear geral univariado, ajustado pelas covariáveis idade, sexo, índice de massa corporal, nível de atividade física e histórico de quedas, utilizando o software SPSS 17.0, com nível de significância de 5\%. Não houve diferença do PT abdutor e adutor do quadril entre os grupos após análise univariada com ajustamento. Observouse que idosos com medo de quedas não apresentam prejuízos na função muscular dos estabilizadores de quadril quando comparados a idosos sem medo de quedas.

Descritores | Torque; Quadril; Equilíbrio; Idoso.
\end{abstract}

ABSTRACT| The fear of falling can cause older adults to develop strategies that alter the semi-static and dynamic balance, predisposing them to increased risk of falling. The muscular function of abductors and adductors plays an important role in maintaining postural stability. However, it is unknown whether older people with fear of falling have greater impairment in hip muscular function. Thus, the objective was to compare the isometric peak torque (PT) of hip abductor and adductor muscles among older adults with and without fear of falling. Participants were divided into two groups: with ( $n=81$ ) and without $(n=81)$ fear of falling. The PT of hip abductors and adductors was obtained with isokinetic dynamometer (System 4 Pro, Biodex, New York, USA). The PT groups were compared using general linear model univariate, adjusted for covariates age, sex, body mass index, physical activity level and history of falls, using the SPSS 17.0 software, with a significance level of 5\%. No difference was found between the PT of hip abductor and adductor and the groups after univariate analysis with adjustment. We observed that older people with fear of falling have no muscle function loss of the hip stabilizers when compared with subjects without fear of falling.

Keywords | Torque; Hip; Balance; Aged.

RESUMEN I El miedo a caerse puede hacer que los ancianos desarrollen estrategias de alteración del equilibrio semiestático y dinámico, predisponiéndolos a un mayor riesgo de caídas. La función muscular de los abductores y de los aductores de cadera juega un papel importante en

Estudo realizado no Laboratório de Avaliação e Reabilitação do Equilíbrio, no Departamento de Ciências da Saúde da Faculdade de Medicina da Universidade de São Paulo (USP), de Ribeirão Preto.

'Universidade de São Paulo (USP) - Ribeirão Preto (SP), Brasil. E-mail: larissabocarde@hotmail.com. Orcid: 0000-0001-6555-1071 2Universidade de São Paulo (USP) - Ribeirão Preto (SP), Brasil. E-mail: jmelloporto@hotmail.com. Orcid: 0000-0002-8128-2083 3 Universidade de São Paulo (USP) - Ribeirão Preto (SP), Brasil. E-mail: renatocfjunior@hotmail.com. Orcid: 0000-0002-9603-981X ${ }^{4}$ Universidade de São Paulo (USP) - Ribeirão Preto (SP), Brasil. E-mail: juliadornof@hotmail.com. Orcid: 0000-0002-9379-5724 ${ }_{5}^{5}$ Universidade de São Paulo (USP) - Ribeirão Preto (SP), Brasil. E-mail: anapaulanakaishi@hotmail.com. Orcid: 0000-0003-2970-0222 ${ }^{6}$ Universidade de São Paulo (USP) - Ribeirão Preto (SP), Brasil. E-mail: dabreu@fmrp.usp.br. Orcid: 0000-0003-4681-2613 
el mantenimiento de la estabilidad postural. Sin embargo, no se sabe si los ancianos con miedo a caerse tienen un mayor deterioro en la función muscular de la cadera. Por lo tanto, el objetivo fue comparar el torque máximo (TM) isométrico de los músculos abductores y aductores de cadera entre los ancianos con miedo y sin miedo a caerse. Los participantes se dividieron en dos grupos: con miedo a caerse $(n=81)$ y $\sin (n=81)$ miedo a caerse. El TM de los abductores y aductores de cadera se obtuvo mediante la utilización del dinamómetro isocinético (System 4 Pro, Biodex, Nueva York, EE.UU.). La comparación del TM de los grupos se realizó mediante un modelo lineal general univariado, ajustado por covariables edad, género, índice de masa corporal, nivel de actividad física e historial de caídas, utilizando el software SPSS 17.0, con nivel de significancia del 5\%. No hubo diferencias entre el TM del abductor y del aductor de cadera entre los grupos tras el análisis univariado con ajuste. Se observó que los ancianos con miedo a caerse no presentaron daños en la función muscular de los estabilizadores de la cadera en comparación con los ancianos sin miedo a caerse.

Palabras clave I Torque; Cadera; Balance; Anciano.

\section{INTRODUÇÃO}

Para mobilidade e funcionalidade, os indivíduos necessitam de múltiplos movimentos articulares gerados pela ativação simultânea de diversos grupos musculares ${ }^{1}$. No entanto, o envelhecimento pode gerar declínios progressivos nos sistemas fisiológicos ${ }^{2}$ que comprometem funções musculoesqueléticas, gerando perdas graduais neuromusculares ${ }^{3}$ e aumentando a propensão às quedas ${ }^{4}$.

Sabe-se que a fraqueza muscular de membros inferiores, decorrente do envelhecimento, é fator predisponente para prejuízos no equilíbrio postural ${ }^{5}$. Dessa forma, os músculos abdutores e adutores de quadril têm importante papel na manutenção da estabilidade pélvica, coordenando movimentos médio-laterais e sustentando o controle postural em base estreita ${ }^{6}$. Apesar disso, no que diz respeito à predisposição a quedas, há controvérsias na literatura em relação à fraqueza desses grupos musculares ${ }^{6-8}$.

Ainda, sendo as quedas eventos multifatoriais em idosos, outros fatores também aumentam o risco de cair, dentre eles, o medo de quedas, que pode fazer com que o indivíduo desenvolva estratégias que alteram o equilíbrio semiestático e dinâmico 9 . O medo de quedas pode resultar de um episódio de queda ou preceder tal evento $^{10}$ e está associado a consequências negativas a longo prazo, como a redução na qualidade de vida, dependência física, depressão e prejuízos na função física e psicossocial ${ }^{11}$.

No que se refere a prejuízos na função física, não se sabe se indivíduos com medo de quedas apresentam prejuízos na função muscular do quadril, o que poderia prejudicar seu equilíbrio postural ${ }^{5,6}$. Assim, o objetivo deste estudo foi comparar o pico de torque isométrico dos abdutores e adutores de quadril entre idosos da comunidade com e sem medo de quedas. A hipótese do presente estudo é que a força muscular dos estabilizadores de quadril é menor no grupo com medo de quedas.

\section{METODOLOGIA}

\section{Sujeitos e desenho do estudo}

Trata-se de um estudo observacional, transversal e com 162 idosos (acima de 60 anos) de ambos os sexos, independentes e autônomos, que foram divididos em dois grupos de acordo com o autorrelato de medo de quedas: grupo com e grupo sem medo de quedas. Os voluntários foram recrutados nos Núcleos de Saúde da Família, na comunidade e em grupos de atividades sociais de Ribeirão Preto (SP). As avaliações ocorreram entre agosto de 2015 a julho de 2016. Foram excluídos idosos com comprometimento cognitivo apontado pelo miniexame do estado mental de acordo com a escolaridade ${ }^{12}$; doença musculoesquelética/neurológica que influenciasse a força muscular do quadril; prótese de joelho e/ou quadril; e indivíduos com doença cardiovascular descompensada que contraindicasse esforço físico.

\section{Procedimentos}

As avaliações ocorreram em dois dias, com intervalo de dois a sete dias entre elas. No primeiro dia, foram coletados os dados para caracterização dos participantes: autorrelato de medo de quedas, peso, altura, IMC (índice de massa corporal), sexo, idade, nível de atividade física e histórico de quedas.

O medo de quedas foi avaliado por meio de questionamento direto "O senhor tem medo de cair?", sendo que os participantes que responderam "Sim, eu 
tenho" se enquadraram no grupo com medo, enquanto aqueles que responderam "Não, não tenho medo" se enquadraram no grupo sem medo de quedas. Durante tal questionamento foi enfatizada a diferença entre medo de cair e cautela durante as atividades.

O questionário internacional de atividade física (IPAQ) foi utilizado para avaliar o nível de atividade física dos participantes ${ }^{13}$, de acordo com a prática de caminhada, atividade moderada ou vigorosa. Assim, os indivíduos foram classificados como tendo baixo, médio ou alto nível de atividade física. Para o histórico de quedas foi considerado o autorrelato do número de quedas ocorrido nos últimos seis meses à avaliação.

Ainda no primeiro dia de coleta foi feita a familiarização do participante em dinamômetro isocinético, sendo o teste repetido no segundo dia para obtenção do pico de torque isométrico (PT) dos abdutores e adutores de quadril.

O teste de força muscular foi realizado no equipamento dinamômetro isocinético (System 4 Pro, Biodex, Nova York, EUA), devidamente calibrado de acordo com recomendações do fabricante. Previamente à avaliação do PT, os participantes realizaram aquecimento em bicicleta ergométrica por cinco minutos. Foi avaliado apenas o membro inferior dominante, sendo definido como membro de escolha para chutar uma bola. Para a obtenção do PT dos abdutores e adutores do quadril, cada participante foi posicionado em decúbito lateral, com o membro a ser testado para cima, com tronco e membro inferior contralateral fixados por cintos. $\mathrm{O}$ eixo do dinamômetro foi alinhado com a intersecção de duas retas: uma medial ao trocânter maior do fêmur e outra longitudinal à espinha ilíaca póstero-superior ${ }^{14}$. $\mathrm{O}$ membro a ser testado foi fixado em uma amplitude de 15 graus de abdução do quadri1 ${ }^{14}$. Para cada grupo muscular, foram realizadas três contrações isométricas voluntárias máximas de cinco segundos de duração e intervalo de um minuto entre elas. A média do PT foi normalizada pela massa corporal do indivíduo $\left(\mathrm{Nm} \cdot \mathrm{kg}^{-1}\right)$, a fim de refletir com maior precisão a função dos músculos em relação à massa do indivíduo ${ }^{15}$.

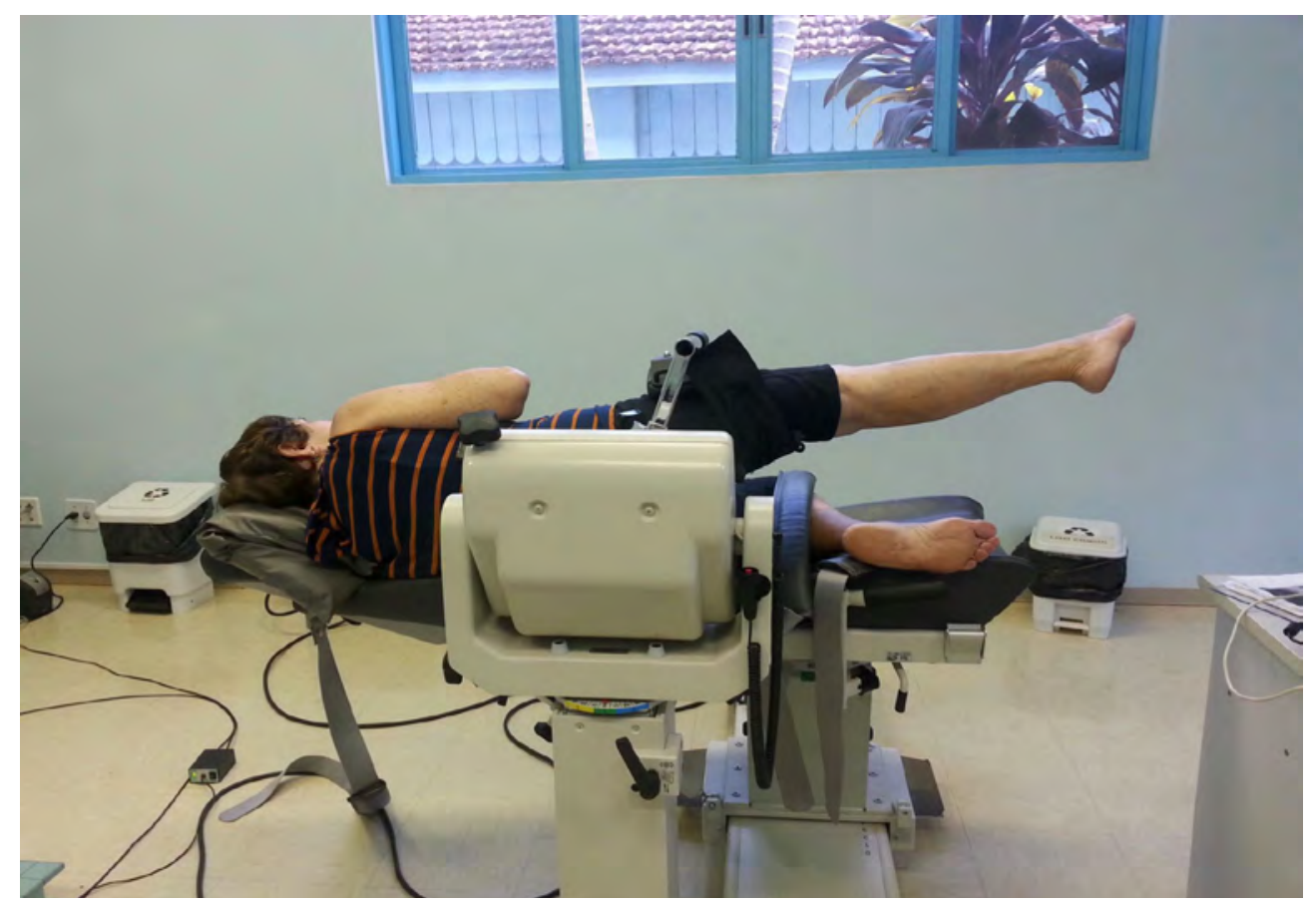

Figura 1. Posicionamento para obtenção do pico de torque isométrico dos abdutores e adutores de quadril no dinamômetro isocinético

\section{Análise estatística}

Para caracterização da amostra foram utilizados médias, desvios-padrão e frequências, conforme apropriado. As comparações das características entre os grupos foram realizadas pelo teste t para as variáveis contínuas, Mann-Whitney para as variáveis ordinais e qui-quadrado para variáveis nominais. Modelo linear geral univariado foi usado para comparações entre a variável independente (medo de quedas) e as variáveis dependentes (pico de torque de adutores e abdutores de quadril). Para amenizar a interferência de variáveis 
de confusão, o modelo estatístico foi ajustado com as seguintes covariáveis: idade, sexo, IMC, nível de atividade física e histórico de quedas. As análises foram desenvolvidas utilizando o software Statistical Package for the Social Sciences (SPSS) 17.0 e foi adotado nível de significância de $5 \%(\mathrm{p} \leq 0,05)$.

\section{RESULTADOS}

A Tabela 1 traz os dados de caracterização da amostra. O grupo com medo de quedas apresentou maior número de mulheres $(\mathrm{p}=0,005)$ e menor altura $(\mathrm{p}=0,019)$ do que o grupo sem medo de cair.

Tabela 1. Características dos participantes com valores descritos em média (desvio-padrão) e frequência absoluta/porcentagem

\begin{tabular}{|c|c|c|c|c|}
\hline & & Com medo de quedas $(n=81)$ & $\begin{array}{l}\text { Sem medo de } \\
\text { quedas }(n=81)\end{array}$ & Valor de $p$ \\
\hline Idade (anos) & & $69,41(5,47)$ & $68,60(5,35)$ & 0,340 \\
\hline Peso (kg) & & $69,13(14,41)$ & $69,55(12,27)$ & 0,804 \\
\hline Altura (m) & & $1,55(0,69)$ & $1,58(0,80)$ & $0,019 *$ \\
\hline IMC $\left(\mathrm{kg} \cdot \mathrm{m}^{-2}\right)$ & & $28,61(5,08)$ & $27,76(5,47)$ & 0,231 \\
\hline Sexo feminino $(n / \%)$ & & $73 / 90,12$ & $59 / 72,83$ & $0,005^{*}$ \\
\hline \multirow[t]{2}{*}{ Histórico de quedas (\%) } & & 37,03 & 25,92 & 0,129 \\
\hline & Baixo & 34,57 & 30,86 & \\
\hline \multirow[t]{2}{*}{ Nível de atividade física (\%) } & Moderado & 59,25 & 62,96 & 0,656 \\
\hline & Alto & 6,18 & 6,18 & \\
\hline
\end{tabular}

* $p \leq 0,05$; IMC: índice de massa corporal.

A Tabela 2 representa a comparação do PT abdutor e adutor de quadril entre os grupos. A análise univariada sem ajustamento mostrou que o grupo sem medo apresentou maior PT dos adutores de quadril em relação ao grupo com medo de quedas $(\mathrm{p}=0,007)$. Porém, quando os dados foram ajustados no modelo, essa diferença entre os grupos não se manteve $(\mathrm{p}=0,129)$.

Tabela 2. Modelo linear geral univariado para comparação do pico de torque de adutores e abdutores de quadril entre os grupos com e sem medo de quedas

\begin{tabular}{|c|c|c|c|c|c|c|}
\hline & \multirow[t]{2}{*}{$\begin{array}{l}\text { Com medo de } \\
\text { quedas }(n=81)\end{array}$} & \multirow[t]{2}{*}{$\begin{array}{l}\text { Sem medo de quedas } \\
\qquad(n=81)\end{array}$} & \multicolumn{2}{|c|}{$\begin{array}{l}\text { Efeito entre os grupos } \\
\text { (valor de } p)^{1}\end{array}$} & \multirow[t]{2}{*}{$\begin{array}{l}\text { Média da } \\
\text { diferença }\end{array}$} & \multirow[t]{2}{*}{$\begin{array}{c}\text { Intervalo de } \\
\text { confiança de } 95 \%\end{array}$} \\
\hline & & & Não ajustado & Ajustado $^{2}$ & & \\
\hline PT abdutores ( $\left.\mathrm{Nm}^{\mathrm{kg}}{ }^{-1}\right)$ & $0,75(0,25)$ & $0,81(0,24)$ & 0,196 & 0,732 & 0,051 & $-0,026-0,129$ \\
\hline PT adutores $\left(\mathrm{Nm}^{\mathrm{kg}} \mathrm{kg}^{-1}\right)$ & $0,60(0,24)$ & $0,71(0,25)$ & 0,007 & 0,129 & 0,108 & $-0,029-0,187$ \\
\hline
\end{tabular}

PT: pico de torque; 'Valores de p na comparação entre os grupos (modelo linear geral); ${ }^{2}$ Ajustamento: idade, sexo, IMC, nível de atividade física e histórico de quedas. Valores descritos em média (desvio-padrão).

\section{DISCUSSÃO}

O presente estudo demonstrou que idosos com medo de quedas não apresentam prejuízos na força muscular dos abdutores e adutores de quadril, quando comparados com idosos sem medo de cair. A hipótese inicial do estudo de que a força muscular de quadril fosse menor no grupo de idosos com medo de quedas foi embasada no fato de que esses idosos estão sujeitos a prejuízos não apenas psicossociais, mas também físicos ${ }^{11}$, e o fato de terem um maior comprometimento dos músculos estabilizadores pélvicos poderia ser uma das causas que aumenta a instabilidade postural e, consequentemente, aumenta a sensação de insegurança ao realizar atividades funcionais rotineiras, sendo reportado pelo idoso como medo de cair.
Entretanto, a piora da função muscular e da capacidade funcional pode ser uma consequência do medo de cair. Nesse sentido, Park et al. ${ }^{12}$ observaram que idosos com medo, independentemente de terem ou não sofrido quedas, obtiveram menor densidade mineral óssea, menor força de preensão palmar e maior tempo na execução do teste timed up and go. Em um estudo prospectivo, que examinou a relação entre medo de quedas e declínio funcional, foi demonstrado que idosos com medo de cair desenvolvem isolamento social, o que causa descondicionamento físico e diminuição da funcionalidade, influenciando negativamente o controle postural e gerando um consequente aumento no risco de quedas ${ }^{9}$. Em casos extremos, o medo de quedas pode induzir excessiva evitação de atividades, o que acarreta redução do nível de atividade física, perda de independência e incapacidade, 
já que está diretamente ligado ao declínio funciona ${ }^{13,14}$. Porém, em nossa amostra, não houve diferença no nível de atividade física entre idosos com e sem medo de quedas, sugerindo que o nível de atividade física, e não o medo de quedas em si, pode ser mais relevante para a função muscular do quadril.

Em relação aos possíveis prejuízos físicos decorrentes da presença de medo de quedas em idosos, poucos estudos avaliam sua influência na função muscular de membros inferiores. Young e Williams ${ }^{15}$ demonstraram que quando os indivíduos com medo de quedas estão expostos a atividades funcionais dinâmicas, desenvolvem uma "estratégia de enrijecimento", reduzindo o deslocamento do centro de massa decorrente de cocontração reflexa dos músculos tibial anterior, gastrocnêmio e sóleo, resultando em menor amplitude de movimento de tornozelo e menor oscilação corporal, aumentando, dessa forma, o risco de quedas. Trombetti et al. ${ }^{16}$ corroboram os achados descritos anteriormente, uma vez que identificaram associação entre massa, força e potência muscular dos extensores de joelho com medo de quedas, independente da presença ou não de restrição de mobilidade. Gadelha et al. ${ }^{17}$ investigaram a associação entre qualidade muscular, equilíbrio, medo de cair e quedas prévias em mulheres idosas da comunidade. Para isso, o medo de quedas foi verificado por meio da escala falls efficacy scale-international (FES-I ${ }^{18}$ e o PT do músculo quadríceps no membro dominante foi avaliado de forma isométrica em dinamômetro isocinético. Além disso, a qualidade muscular foi definida pela fórmula: PT $(\mathrm{Nm})$ /espessura muscular $(\mathrm{mm})$, sendo que os indivíduos que obtiveram valores $\leq 3,6 \mathrm{Nm} . \mathrm{mm}^{-1}$ foram considerados como tendo baixa qualidade muscular. Em relação a essas variáveis, os autores concluíram que há associação entre a qualidade muscular e medo de quedas, sendo que a mensuração da qualidade muscular do quadríceps é um importante componente para avaliar risco de quedas em idosos da comunidade.

Apesar dessas evidências, não foram encontrados estudos que investigaram propriamente a força muscular de quadril em idosos com medo de quedas. Uma vez que os músculos abdutores e adutores de quadril são responsáveis pela manutenção da estabilidade pélvica e da coordenação de movimentos médio-laterais, sustentando o controle postural em base estreita ${ }^{6,19}$, a hipótese inicial do presente estudo era que idosos com medo de cair pudessem apresentar prejuízos nesses grupos musculares, o que poderia explicar, em parte, o risco aumentado de quedas a que estão expostos ${ }^{9,15}$. Apesar de no presente estudo essa diferença não ter sido encontrada, é possível que outros fatores musculoesqueléticos interfiram no controle postural de idosos com medo de quedas, como a cocontração dos músculos do quadril quando os indivíduos são expostos a uma perturbação ou mesmo prejuízos em outros parâmetros, como potência muscular.

Este estudo apresenta algumas limitações: a avaliação da força muscular do quadril foi feita de forma isométrica, sendo que a análise da força muscular dos abdutores e adutores de quadril, realizada de forma dinâmica e funcional, poderia proporcionar mais informações a respeito da função muscular do quadril em idosos com medo de quedas. Além disso, a amostra não continha idosos acima de 80 anos, o que restringiu o estudo a idosos mais jovens. Ainda, a maior parte da amostra de ambos os grupos foi composta de idosos fisicamente ativos (nível moderado de atividade física), sendo que investigar a influência do medo de quedas em uma amostra de idosos sedentários poderá trazer informações adicionais ao presente estudo.

\section{CONCLUSÃO}

Os resultados deste estudo sugerem que idosos jovens com medo de quedas não apresentam prejuízos na força muscular dos abdutores e adutores de quadril, quando comparados com idosos sem medo de cair.

\section{REFERÊNCIAS}

1. Allison SJ, Brooke-Wavell K, Folland JP. Multiple joint muscle function with ageing: the force-velocity and power-velocity relationships in young and older men. Aging Clin Exp Res. 2013;25(2):159-66. doi: 10.1007/s40520-013-0024-y

2. Edwén CE, Thorlund JB, Magnusson SP, Slinde F, Svantesson $U$, Hulthén L, et al. Stretch-shortening cycle muscle power in women and men aged 18-81 years: influence of age and gender. Scand J Med Sci Sport. 2014;24(4):717-26. doi: 10.1111/sms.12066

3. Barbieri E, Agostini D, Polidori E, Potenza L, Guescini M, Lucertini F, et al. The pleiotropic effect of physical exercise on mitochondrial dynamics in aging skeletal muscle. Oxid Med Cell Longev. 2015;1:1-15. doi: 10.1155/2015/917085

4. Bento PC, Pereira G, Ugrinowitsch C, Rodacki AL. Peak torque and rate of torque development in elderly with and without fall history. Clin Biomech. 2010;25(5):450-4. doi: 10.1016/ j.clinbiomech.2010.02.002

5. Hashish R, Samarawickrame SD, Wang MY, Yu SS, Salem GJ. The association between unilateral heel-rise performance with static and dynamic balance in community dwelling older adults. Geriatr Nurs. 2015;36(1):30-4. doi: 10.1016/j.gerinurse.2014.09.003 
6. Chang SHJ, Mercer VS, Giuliani CA, Sloane PD. Relationship between hip abductor rate of force development and mediolateral stability in older adults. Arch Phys Med Rehabil. 2005;86(9):1843-50. doi: 10.1016/j.apmr.2005.03.006

7. Pinho L, Dias RC, Souza TR, Freire MTF, Tavares CF, Dias JMD. Avaliação isocinética da função muscular do quadril e do tornozelo em idosos que sofrem quedas. Rev Bras Fisioter. 2005:9(1):93-9.

8. Morcelli MH, Rossi DM, Karuka AH, Crozara LF, Hallal CZ, Marques NR, et al. Peak torque, reaction time, and rate of torque development of hip abductors and adductors of older women. Physiother Theory Pract. 2016;32(1):45-52. doi: 10.3109/09593985.2015.1091870

9. Choi K, Jeon GS, Cho SI. Prospective study on the impact of fear of falling on functional decline among community dwelling elderly women. Int J Environ Res Public Heal. 2017;14(5):E469. doi: 10.3390/ijerph14050469

10. Legters K. Fear of falling. Phys Ther. 2002;82(3):264-72. doi:10.1093/ptj/82.3.264

11. Scheffer AC, Schuurmans MJ, van Dijk N, van der Hooft T, de Rooij SE. Fear of falling: measurement strategy, prevalence, risk factors and consequences among older persons. Age Ageing. 2008;37(1):19-24. doi: 10.1093/ageing/afm169

12. Park JH, Cho H, Shin JH, Kim T, Park SB, Choi BY, et al. Relationship among fear of falling, physical performance, and physical characteristics of the rural elderly. Am J Phys Med Rehabil. 2014;93(5):379-86. doi: 10.1097/PHM.0000000000000009
13. Visschedijk JH, Caljouw MA, Bakkers E, van Balen R, Achterberg WP. Longitudinal follow-up study on fear of falling during and after rehabilitation in skilled nursing facilities. BMC Geriatr. 2015;15(1):161. doi: 10.1186/s12877-015-0158-1

14. Hadjistavropoulos T, Delbaere K, Fitzgerald TD. Reconceptualizing the role of fear of falling and balance confidence in fall risk. J Aging Heal. 2011;23(1):3-23. doi: 10.1177/0898264310378039

15. Young WR, Williams AM. How fear of falling can increase fall-risk in older adults: applying psychological theory to practical observations. Gait Posture. 2015;41(1):7-12. doi: 10.1016/ j.gaitpost.2014.09.006

16. Trombetti A, Reid KF, Hars M, Herrmann FR, Pasha E, Phillips EM, et al. Age-associated declines in muscle mass, strength, power, and physical performance: impact on fear of falling and quality of life. Osteoporos Int. 2016;27(2):463-71. doi: 10.1007/s00198-015-3236-5

17. Gadelha AB, Neri SGR, Nóbrega OT, Pereira JC, Bottaro M, Fonsêca $A$, et al. Muscle quality is associated with dynamic balance, fear of falling, and falls in older women. Exp Gerontol. 2018;104:1-6. doi: 10.1016/j.exger.2018.01.003

18. Yardley L, Beyer N, Hauer K, Kempen G, Piot-Ziegler C, Todd C. Development and initial validation of the Falls Efficacy Scale-International (FES-I). Age Ageing. 2005;34(6):614-9. doi: 10.1093/ageing/afi196

19. Johnson ME, Mille ML, Martinez KM, Crombie G, Rogers MW. Age-related changes in hip abductor and adductor joint torques. Arch Phys Med Rehabil. 2004;85(4):593-7. doi: 10.1016/ j.apmr.2003.07.022 